\title{
A Versatile Synthesis of Fluorinated Uracils in Solution and on Solid-Phase.
}

\author{
Santos Fustero, ${ }^{\star}$ Julio Piera, Juan F. Sanz-Cervera, ${ }^{\star}$ \\ Silvia Catalán, and Carmen Ramírez de Arellano. \\ Departamento de Química Orgánica. Universidad de Valencia. E-46100 Burjassot, Spain. \\ santos.fustero@uv.es; juan.f.sanz@uv.es
}

\section{SUPPORTING INFORMATION}

\section{Preparation of compounds 8.}

General procedure. $\mathrm{N}$-butyllithium (10.5 mmol, $2.5 \mathrm{M}$ in hexane) was slowly added to a solution of diisopropylamine $(10.5 \mathrm{mmol})$ in $\mathrm{THF}(15 \mathrm{~mL})$ at $-30^{\circ} \mathrm{C}$. The mixture was stirred at that temperature for $30 \mathrm{~min}$, after which time the temperature was lowered to $-50{ }^{\circ} \mathrm{C}$. A solution of the ester $(8.4 \mathrm{mmol})$ in THF $(10 \mathrm{~mL})$ was then added dropwise and the reaction mixture was stirred until it took on a yellow color, which indicated the complete formation of the enolate. The temperature was then lowered to $-78{ }^{\circ} \mathrm{C}$ and a solution of the nitrile $(7.0 \mathrm{mmol})$ in THF $(10 \mathrm{~mL})$ was slowly added. The progress of the reaction was monitored by means of TLC, and after $c a .1-1.5 \mathrm{~h}$ it was quenched with satd. aq. $\mathrm{NH}_{4} \mathrm{Cl}$ solution and extracted with $\mathrm{AcOEt}(3 \times 20 \mathrm{~mL})$. The organic layers were pooled together, washed with brine, dried over anh. $\mathrm{Na}_{2} \mathrm{SO}_{4}$, and concentrated to give crude product 8 , which was purified in each case as described below.

Ethyl 3-amino-4,4-difluoro-4-phenyl-2-butenoate (8a). Flash chromatography of the crude reaction product [ $n$-hexane:EtOAc (4:1)] on deactivated silica gel (2\% $\mathrm{Et}_{3} \mathrm{~N}$ in hexane) gave a yellow oil (97\%). ${ }^{1} \mathrm{H}$ NMR $\left(300 \mathrm{MHz}, \mathrm{CDCl}_{3}\right) \delta 1.15$ (t, $\left.J=7.2 \mathrm{~Hz}, 2 \mathrm{H}\right), 4.03$ (c, $\left.J=7.6 \mathrm{~Hz}, 2 \mathrm{H}\right), 4.82$ (s, 1H), 7.30-7.35 (m, 3H), 7.45-7.48 (m, 2H); $\left.{ }^{13} \mathrm{C} \mathrm{NMR} \mathrm{(75.5} \mathrm{MHz,} \mathrm{CDCl}_{3}\right) \delta 13.3(\mathrm{c}), 58.4(\mathrm{t}), 84.6(\mathrm{t}$, $\left.{ }^{3} J_{\mathrm{CF}}=5.5 \mathrm{~Hz}\right), 116.6\left(\mathrm{t},{ }^{1} J_{\mathrm{CF}}=245.4 \mathrm{~Hz}\right), 124.4\left(\mathrm{t},{ }^{3} J_{\mathrm{CF}}=5.7 \mathrm{~Hz}\right), 127.6(\mathrm{~d}), 129.7(\mathrm{~d}), 133.4\left(\mathrm{t},{ }^{2} J_{\mathrm{CF}}=\right.$ $27.0 \mathrm{~Hz}), 154.2\left(\mathrm{t},{ }^{2} J_{\mathrm{CF}}=28.7 \mathrm{~Hz}\right), 168.7(\mathrm{~s}) ;{ }^{19} \mathrm{~F}$ NMR $\left(282 \mathrm{MHz}, \mathrm{CDCl}_{3}\right) \delta-99.8(\mathrm{~s}, 2 \mathrm{~F}) . \mathrm{HRMS}$ calcd for $\mathrm{C}_{12} \mathrm{H}_{13} \mathrm{~F}_{2} \mathrm{NO}_{2} 241.0914$, found 241.0902. 
Ethyl 3-amino-4,4-difluoro-4-phenyl-2-methyl-2-butenoate (8b). Flash chromatography of the crude reaction product [ $n$-hexane:EtOAc (4:1)] on deactivated silica gel ( $2 \% \mathrm{Et}_{3} \mathrm{~N}$ in hexane) gave a yellow oil (87\% yield). ${ }^{1} \mathrm{H}$ NMR $\left(300 \mathrm{MHz} \mathrm{CDCl}_{3}\right) \delta 1.21(\mathrm{t}, J=7.2 \mathrm{~Hz}, 3 \mathrm{H}), 1.45(\mathrm{t}, J=2.2 \mathrm{~Hz}$, $3 \mathrm{H}), 4.10(\mathrm{c}, J=7.1 \mathrm{~Hz}, 2 \mathrm{H}), 6.79(\mathrm{br} \mathrm{s}, 2 \mathrm{H}), 7.36-7.41(\mathrm{~m}, 3 \mathrm{H}), 7.51-7.55(\mathrm{~m}, 2 \mathrm{H}) ;{ }^{13} \mathrm{C}$ NMR $(75.5$ $\left.\mathrm{MHz}_{\mathrm{CDCl}}\right) \delta 12.5(\mathrm{c}), 14.8(\mathrm{c}), 60.2(\mathrm{t}), 92.8\left(\mathrm{t},{ }^{3} J_{\mathrm{CF}}=2.9 \mathrm{~Hz}\right), 118.7\left(\mathrm{t},{ }^{1} J_{\mathrm{CF}}=244.3 \mathrm{~Hz}\right), 125.9(\mathrm{t}$, $\left.{ }^{3} J_{\mathrm{CF}}=5.2 \mathrm{~Hz}\right), 129.0(\mathrm{~d}), 131.1\left(\mathrm{t},{ }^{4} J_{\mathrm{CF}}=2.0 \mathrm{~Hz}\right), 135.3\left(\mathrm{t},{ }^{2} J_{\mathrm{CF}}=26.4 \mathrm{~Hz}\right), 151.4\left(\mathrm{t},{ }^{2} J_{\mathrm{CF}}=24.7 \mathrm{~Hz}\right)$, 171.5 (s); ${ }^{19} \mathrm{~F}$ NMR (282 MHz, $\left.\mathrm{CDCl}_{3}\right) \delta-92.3$ (s, 2F). HRMS calcd for $\mathrm{C}_{13} \mathrm{H}_{15} \mathrm{~F}_{2} \mathrm{NO}_{2} 255.1071$, found 255.1078 .

Ethyl 3-amino-4,4-difluoro-4-phenyl-2-phenyl-2-butenoate (8c). Flash chromatography of the crude reaction product $\left[n\right.$-hexane:EtOAc (4:1)] on deactivated silica gel ( $2 \% \mathrm{Et}_{3} \mathrm{~N}$ in hexane) gave a yellow oil (93\% yield). ${ }^{1} \mathrm{H}$ NMR $\left(300 \mathrm{MHz} \mathrm{CDCl}_{3}\right) \delta 1.12(\mathrm{t}, J=7.2,3 \mathrm{H}), 4.17(\mathrm{c}, J=7.0,2 \mathrm{H})$, 7.40-7.48 (m, 3H), 7.69-7.90 (m, 2H), 7.98 (br s, 1H), 8.15 (br s, 1H); $\left.{ }^{13} \mathrm{C} \mathrm{NMR} \mathrm{(75.5} \mathrm{MHz,} \mathrm{CDCl}_{3}\right) \delta$ $13.3(\mathrm{c}), 58.4(\mathrm{t}), 84.6\left(\mathrm{t},{ }^{3} J_{\mathrm{CF}}=5.5\right), 116.6\left(\mathrm{t},{ }^{1} J_{\mathrm{CF}}=245.4\right), 124.4\left(\mathrm{t},{ }^{3} J_{\mathrm{CF}}=5.7\right), 127.6(\mathrm{~d}), 129.7(\mathrm{~d})$, $133.4\left(\mathrm{t},{ }^{2} J_{\mathrm{CF}}=27.0\right), 154.2\left(\mathrm{t},{ }^{2} J_{\mathrm{CF}}=28.7\right), 168.7(\mathrm{~s}) ;{ }^{19} \mathrm{~F}$ NMR $\left(282 \mathrm{MHz}, \mathrm{CDCl}_{3}\right) \delta-96.2(\mathrm{~s}, 2 \mathrm{~F})$. HRMS calcd for $\mathrm{C}_{18} \mathrm{H}_{17} \mathrm{~F}_{2} \mathrm{NO}_{2} 317.1227$, found 317.1209.

Ethyl 3-amino-4,4-difluoro-4-(1-naphtyl)-2-phenyl-2-butenoate (8d). Flash chromatography of the crude reaction product [ $n$-hexane:EtOAc (4:1)] on deactivated silica gel ( $2 \% \mathrm{Et}_{3} \mathrm{~N}$ in hexane) gave a yellow oil (70\% yield). ${ }^{1} \mathrm{H}$ NMR $\left(300 \mathrm{MHz}, \mathrm{CDCl}_{3}\right) \delta 1.12(\mathrm{t}, J=7.2,3 \mathrm{H}), 4.17(\mathrm{c}, J=7.0$, 2H), 7.40-7.48 (m, 3H), 7.69-7.90 (m, 2H), 7.98 (br s, 1H), 8.15 (br s, 1H); ${ }^{13} \mathrm{C}$ NMR (75.5 MHz, $\left.\mathrm{CDCl}_{3}\right) \delta 14.3(\mathrm{c}), 59.7(\mathrm{t}), 85.1\left(\mathrm{t},{ }^{3} J_{\mathrm{CF}}=5.1\right), 115.9\left(\mathrm{t},{ }^{1} J_{\mathrm{CF}}=244.7\right), 122.6\left(\mathrm{t},{ }^{4} J_{\mathrm{CF}}=3.1\right), 123.3(\mathrm{~d})$, $125.2\left(\mathrm{t},{ }^{3} J_{\mathrm{CF}}=9.5\right), 125.2\left(\mathrm{t},{ }^{2} J_{\mathrm{CF}}=21.9\right), 125.6(\mathrm{~d}), 127.1(\mathrm{~d}), 128.0(\mathrm{~s}), 128,6(\mathrm{~d}), 131.6(\mathrm{~d}), 132.2$ $(\mathrm{s}), 147.8\left(\mathrm{t},{ }^{2} J_{\mathrm{CF}}=29.6\right), 163.2(\mathrm{~s}) ;{ }^{19} \mathrm{~F} \mathrm{NMR}\left(282 \mathrm{MHz}, \mathrm{CDCl}_{3}\right) \delta-96.2(\mathrm{~s}, 2 \mathrm{~F})$. HRMS calcd for (M1) $\mathrm{C}_{16} \mathrm{H}_{14} \mathrm{~F}_{2} \mathrm{NO}_{2} 290.0992$, found 290.1008 .

Ethyl 3-amino-4,4,5,5,6,6,7,7,8,8,9,9,10,10,10-pentadecafluoro-dec-2-enoate (8e). Flash chromatography of the crude reaction product [ $n$-hexane:EtOAc $(4: 1)]$ on deactivated silica gel $(2 \%$ $\mathrm{Et}_{3} \mathrm{~N}$ in hexane) gave a colorless oil (91\% yield). ${ }^{1} \mathrm{H} \mathrm{NMR}\left(300 \mathrm{MHz}, \mathrm{CDCl}_{3}\right) \delta 1.16(\mathrm{t}, J=7.1,3 \mathrm{H})$, $4.06(\mathrm{c}, J=7.1,2 \mathrm{H}), 4.97(\mathrm{~s}, 1 \mathrm{H}), 6.30(\mathrm{br} \mathrm{s}, 2 \mathrm{H}) ;{ }^{13} \mathrm{C} \mathrm{NMR}\left(75.5 \mathrm{MHz}, \mathrm{CDCl}_{3}\right) \delta 14.0(\mathrm{c}), 60.0(\mathrm{t})$, $88.5\left(\mathrm{t},{ }^{3} J_{\mathrm{CF}}=6.3\right), 104.3-123.6$ (the signals from the $\mathrm{C}_{7} \mathrm{~F}_{15}$ group were obscured due to their low intensity), $147.3\left(\mathrm{t},{ }^{2} J_{\mathrm{CF}}=24.4\right), 169.3(\mathrm{~s}) ;{ }^{19} \mathrm{~F} \mathrm{NMR}\left(282 \mathrm{MHz}, \mathrm{CDCl}_{3}\right) \delta-82.1-(-82.2)(\mathrm{m}, 3 \mathrm{H})$, 119.5-(-119.6) (m, 2H), -122.3-(-122.5) (m, 2H), 122.9 (m, 2H), 123.3-(-123.4) (m, 2H), 123.6-(123.7) (m, 2H), 127.1-(-127.3) (m, 2H). HRMS calcd for $\mathrm{C}_{12} \mathrm{H}_{8} \mathrm{~F}_{15} \mathrm{NO}_{2} 483.0315$, found 483.0245. 


\section{Preparation of compounds 5.}

General procedure. $\mathrm{NaH}(3.6 \mathrm{mmol})$ was slowly added to a solution of $\beta$-enaminoester 8 (2.0 $\mathrm{mmol})$ in DMF $(3 \mathrm{ml})$ at $0^{\circ} \mathrm{C}$. The mixture was stirred at that temperature for $30 \mathrm{~min}$, after which time the isocyanate or isotiocyanate $(2.4 \mathrm{mmol})$ was added. The mixture was then stirred at room temperature. The progress of the reaction was monitored by means of TLC and after $c a .30 \mathrm{~min}$ it was quenched with $\mathrm{H}_{2} \mathrm{O}$ and the solvent was removed under vacuum. The resulting crude mixture was taken up in $\mathrm{H}_{2} \mathrm{O}(10 \mathrm{ml})$ and then extracted with $\mathrm{CH}_{2} \mathrm{Cl}_{2}(3 \times 10 \mathrm{ml})$. The uracils and tiouracils 8 proved to be soluble in $\mathrm{H}_{2} \mathrm{O}$ and insoluble in $\mathrm{CH}_{2} \mathrm{Cl}_{2}$. The aqueous layer was concentrated to a volume of $2 \mathrm{ml}$ and then extracted with AcOEt $(3 \times 10 \mathrm{ml})$. The organic layers were pooled together, dried over anh. $\mathrm{Na}_{2} \mathrm{SO}_{4}$, and concentrated to give crude product 5 , which was purified in each case as described below.

6-(Difluoromethylphenyl)-3-(4-fluorophenyl)-2,4-pyrimidindione (5a). The crude reaction product was washed with $n$-hexane- $\mathrm{CH}_{2} \mathrm{Cl}_{2}$ (5:1) to give pure 5a as a white solid (90\% yield): $\mathrm{mp}$ 166-168 ${ }^{\circ} \mathrm{C} .{ }^{1} \mathrm{H}$ NMR $\left(300 \mathrm{MHz}, \mathrm{CDCl}_{3}\right) \delta 5.87$ (s, $\left.1 \mathrm{H}\right), 7.04-7.07$ (m, 4H), 7.32-7.35 (m, 3H), 7.52$7.55(\mathrm{~m}, 2 \mathrm{H}) ;{ }^{13} \mathrm{C} \operatorname{NMR}\left(75.5 \mathrm{MHz}, \mathrm{CDCl}_{3}\right) \delta 95.8\left(\mathrm{t},{ }^{3} J_{\mathrm{CF}}=5.2\right), 117.1\left(\mathrm{~d},{ }^{2} J_{\mathrm{CF}}=23.0\right), 119.7(\mathrm{t}$, $\left.{ }^{1} J_{\mathrm{CF}}=244.0\right), 127.2\left(\mathrm{t},{ }^{3} J_{\mathrm{CF}}=6.0\right), 129.8(\mathrm{~d}), 131.5(\mathrm{~d}), 132.0\left(\mathrm{~d},{ }^{3} J_{\mathrm{CF}}=9.2\right), 135.2\left(\mathrm{~d},{ }^{4} J_{\mathrm{CF}}=2.8\right), 138.0(\mathrm{t}$, $\left.{ }^{2} J_{\mathrm{CF}}=27.3\right), 161.9(\mathrm{~s}), 163.8\left(\mathrm{~d},{ }^{1} J_{\mathrm{CF}}=244.8\right), 164.8\left(\mathrm{t},{ }^{2} J_{\mathrm{CF}}=29.6\right), 169.7(\mathrm{~s}) ;{ }^{19} \mathrm{~F}$ NMR $(282 \mathrm{MHz}$, $\left.\mathrm{CDCl}_{3}\right) \delta$-101.6 (s, 2F), -117.4 (m, 1F). HRMS calcd for $\mathrm{C}_{17} \mathrm{H}_{11} \mathrm{~F}_{3} \mathrm{~N}_{2} \mathrm{O}_{2} 332.0772$, found 332.0760.

6-(Difluoromethylphenyl)-3-(4-metoxyphenyl)-2,4-pyrimidindione (5b). The crude reaction product was washed with $n$-hexane- $\mathrm{CH}_{2} \mathrm{Cl}_{2}$ (5:1) to give pure $\mathbf{5 b}$ as a white solid (84\% yield): $\mathrm{mp}$ $175-177^{\circ} \mathrm{C} .{ }^{1} \mathrm{H}$ NMR $\left(300 \mathrm{MHz}, \mathrm{CDCl}_{3}\right) \delta 3.70$ (s, 3H), 5.88 (s, 1H), 6.88 (d, $\left.J=9.0,2 \mathrm{H}\right), 6.97(\mathrm{~d}, J=$ 9.0, 2H), 7.34-7.36 (m, 3H), 7.54-7.57 (m, 2H); ${ }^{13} \mathrm{C} \mathrm{NMR}\left(75.5 \mathrm{MHz}, \mathrm{CDCl}_{3}\right) \delta 56.3(\mathrm{c}), 96.1\left(\mathrm{t},{ }^{3} J_{\mathrm{CF}}\right.$ $=4.9), 115.8$ (d), $119.6\left(\mathrm{t},{ }^{1} J_{\mathrm{CF}}=244.0\right), 127.2\left(\mathrm{t},{ }^{3} J_{\mathrm{CF}}=6.0\right), 129.8$ (d), 130.9 (d), 131.5 (d), 131.6 (d), $137.9\left(\mathrm{t},{ }^{2} J_{\mathrm{CF}}=27.3\right), 161.0(\mathrm{~s}), 161.7(\mathrm{~s}), 163.6\left(\mathrm{t},{ }^{2} J_{\mathrm{CF}}=29.9\right), 169.7(\mathrm{~s}) ;{ }^{19} \mathrm{~F} \mathrm{NMR}\left(282 \mathrm{MHz}, \mathrm{CDCl}_{3}\right)$ $\delta$-101.4 (s, 2F). HRMS calcd for $\mathrm{C}_{18} \mathrm{H}_{14} \mathrm{~F}_{2} \mathrm{~N}_{2} \mathrm{O}_{3} 344.0972$, found 344.0987.

6-(Difluoromethylphenyl)-3-(3-trifluorometylphenyl)-2,4-pyrimidindione (5c). The crude reaction product was washed with $n$-hexane- $\mathrm{CH}_{2} \mathrm{Cl}_{2}(5: 1)$ to give pure $\mathbf{5 c}$ as a white solid (76\% yield): mp 165-167 ${ }^{\circ} \mathrm{C} .{ }^{1} \mathrm{H}$ NMR $\left(300 \mathrm{MHz}, \mathrm{CDCl}_{3}\right) \delta 5.89$ (s, $\left.1 \mathrm{H}\right), 7.35-7.49(\mathrm{~m}, 5 \mathrm{H}), 7.50-7.57(\mathrm{~m}, 4 \mathrm{H}) ;{ }^{13} \mathrm{C}$ $\operatorname{NMR}\left(75.5 \mathrm{MHz}, \mathrm{CDCl}_{3}\right) \delta 97.9\left(\mathrm{t},{ }^{3} J_{\mathrm{CF}}=5.2\right), 119.2\left(\mathrm{t},{ }^{1} J_{\mathrm{CF}}=244.5\right), 125.7\left(\mathrm{c},{ }^{1} J_{\mathrm{CF}}=271.3\right), 126.4(\mathrm{c}$, 
$\left.{ }^{3} J_{\mathrm{CF}}=4.9\right), 127.2\left(\mathrm{t},{ }^{3} J_{\mathrm{CF}}=6.0\right), 127.4\left(\mathrm{c},{ }^{3} J_{\mathrm{CF}}=3.8\right), 130.1(\mathrm{~d}), 131.4(\mathrm{~d}), 132.1(\mathrm{~d}), 132.9\left(\mathrm{c},{ }^{2} J_{\mathrm{CF}}=\right.$ 32.5), $134.2(\mathrm{~d}), 136.7\left(\mathrm{t},{ }^{2} J_{\mathrm{CF}}=26.7\right), 138.9(\mathrm{~s}), 158.1(\mathrm{~s}), 159.1\left(\mathrm{t},{ }^{2} J_{\mathrm{CF}}=30.7\right), 167.7(\mathrm{~s}) ;{ }^{19} \mathrm{~F}$ NMR $\left(282 \mathrm{MHz}, \mathrm{CDCl}_{3}\right) \delta-64.4(\mathrm{~s}, 3 \mathrm{~F}),-101.0$ (s, 2F). HRMS calcd for $\mathrm{C}_{18} \mathrm{H}_{11} \mathrm{~F}_{5} \mathrm{~N}_{2} \mathrm{O}_{2}$ 382.0740, found 382.0749 .

6-(Difluoromethylphenyl)-3-(3-trifluorometylphenyl)-2,4-pyrimidindione (5d). The crude reaction product was washed with $n$-hexane- $\mathrm{CH}_{2} \mathrm{Cl}_{2}(5: 1)$ to give pure $\mathbf{5 d}$ as a white solid (86\% yield): mp 214-216 ${ }^{\circ} \mathrm{C} .{ }^{1} \mathrm{H}$ NMR $\left(300 \mathrm{MHz}, \mathrm{CDCl}_{3}\right) \delta 6.03(\mathrm{~s}, 1 \mathrm{H}), 7.25-7.28(\mathrm{~m}, 2 \mathrm{H}), 7.36-7.46(\mathrm{~m}, 3 \mathrm{H})$, 7.55-7.58 (m, 3H), 7.74-7.76 (m, 2H); ${ }^{13} \mathrm{C}$ NMR $\left(75.5 \mathrm{MHz}, \mathrm{CDCl}_{3}\right) \delta 99.1\left(\mathrm{t},{ }^{3} J_{\mathrm{CF}}=5.2\right), 116.9(\mathrm{t}$, $\left.{ }^{1} J_{\mathrm{CF}}=245.7\right), 126.1\left(\mathrm{t},{ }^{3} J_{\mathrm{CF}}=5.7\right), 128.5$ (d), 129.1 (d), 129.2 (d), 129.3 (d), 131.8 (d), $133.5\left(\mathrm{t},{ }^{2} J_{\mathrm{CF}}=\right.$ 26.7), $135.6(\mathrm{~s}), 148.0\left(\mathrm{t},{ }^{2} J_{\mathrm{CF}}=33.0\right), 151.6(\mathrm{~s}), 162.8(\mathrm{~s}) ;{ }^{19} \mathrm{~F} \mathrm{NMR}\left(282 \mathrm{MHz}, \mathrm{CDCl}_{3}\right) \delta-97.3(\mathrm{~s}$, 2F). HRMS calcd for $\mathrm{C}_{17} \mathrm{H}_{12} \mathrm{~F}_{2} \mathrm{~N}_{2} \mathrm{O}_{2} 314.0866$, found 314.0876 .

3-sec-Butyl-6-(difluoromethylphenyl)-2,4-pyrimidindione (5e). Flash chromatography [n-hexane:EtOAc (2:1)] of the crude reaction mixture on silica gel gave $\mathbf{5 e}$ as a white solid (71\% isolated yield): $\mathrm{mp} 116-118^{\circ} \mathrm{C} .{ }^{1} \mathrm{H} \mathrm{NMR}\left(300 \mathrm{MHz}, \mathrm{CDCl}_{3}\right) \delta 0.72(\mathrm{t}, J=7.4,3 \mathrm{H}), 1.30(\mathrm{~d}, J=7.0$, $3 \mathrm{H}), 1.58-1.72(\mathrm{~m}, 1 \mathrm{H}), 1.89-2.05(\mathrm{~m}, 1 \mathrm{H}), 4.69-4.61(\mathrm{~m}, 1 \mathrm{H}), 5.64(\mathrm{~s}, 1 \mathrm{H}), 7.39-7.54(\mathrm{~m}, 5 \mathrm{H}) ;{ }^{13} \mathrm{C}$ NMR (75.5 MHz, $\left.\mathrm{CDCl}_{3}\right) \delta 11.9$ (c), 17.9 (c), 27.2 (t), 52.9 (d), 100.9 (d), 118.2 (t, ${ }^{1} J_{\mathrm{CF}}=244.8$ ), $127.1\left(\mathrm{t},{ }^{3} J_{\mathrm{CF}}=6.0\right), 130.5(\mathrm{~d}), 132.9(\mathrm{~d}), 134.8\left(\mathrm{t},{ }^{2} J_{\mathrm{CF}}=26.4\right), 149.4\left(\mathrm{t},{ }^{2} J_{\mathrm{CF}}=32.7\right), 153.1(\mathrm{~s}), 165.9$ (s); ${ }^{19} \mathrm{~F}$ NMR (282 MHz, $\left.\mathrm{CDCl}_{3}\right) \delta-100.2$ (s, 2F). HRMS calcd for $\mathrm{C}_{15} \mathrm{H}_{16} \mathrm{~F}_{2} \mathrm{~N}_{2} \mathrm{O}_{2} 294.1179$, found 294.1190.

The structure of compound $5 \mathrm{e}$ was determined by means of single crystal X-ray diffraction techniques. The structure shows three independent molecules in the asymmetric unit with the secbutyl group bonded to the N(1) atom (Figures 1 and 3). The heterocyclic ring is planar for all three independent molecules (mean deviation 0.004-0.016). $\mathrm{N}-\mathrm{H} \cdots \mathrm{O}=\mathrm{C}$ hydrogen bonds are present in the crystal packing. Crystal data for 5 e: $\mathrm{C}_{15} \mathrm{H}_{16} \mathrm{~F}_{2} \mathrm{~N}_{2} \mathrm{O}_{2}, M=294.11$, colorless prism of $0.40 \times 0.32 \times 0.10$ mm size, triclinic, $\mathrm{P}-1, \mathrm{a}=11.2061(2), \mathrm{b}=111.3867(2), \mathrm{c}=18.0052(3) \AA ⿻$ $\alpha=106.934(1), \beta=$ 106.934(1), $\gamma=106.934(1), \mathrm{V}=2151.70(6) \AA 3, \mathrm{Z}=6, \mathrm{Dc}=1.363 \mathrm{gcm}-3, \theta \max =30.0^{\circ}$, diffractometer Kappa CCD, Mo Ka $(\lambda=0.71073 \AA)$, $\omega$-scan, $\mathrm{T}=173(2) \mathrm{K}, 9964$ reflections collected of which 8759 were independent $($ Rint $=0.025)$, direct primary solution and refinement on F2 (SHELXL-97, G.M. Sheldrick, University of Göttingen, 1997), 637 refined parameters, free NH, other hydrogen atoms riding, the sec-Bu group of two independent molecules is disordered over two sites, $\mathrm{R} 1[\mathrm{I}>2 \sigma(\mathrm{I})]=0.0630, \mathrm{wR} 2$ (all data) $=0.2055$. 


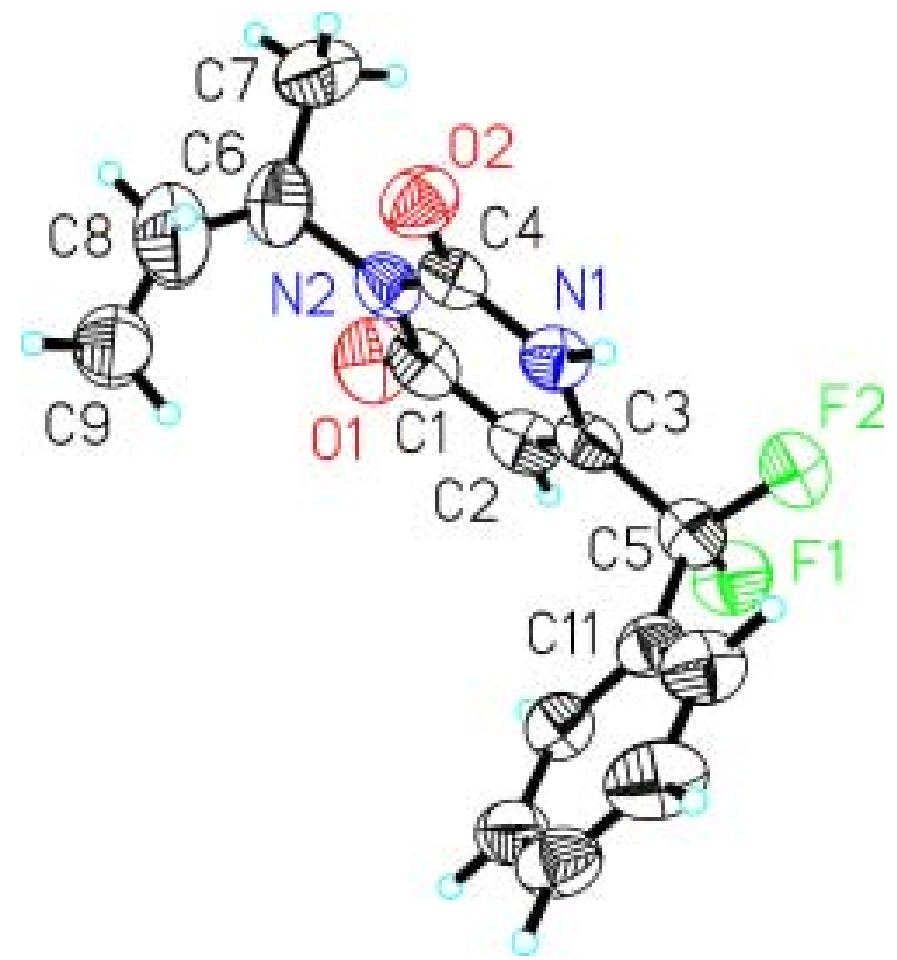

Figure 3. Ellipsoid plot of compound 5e (50\% probability level). See Figure 1 in the main text for an additional view.

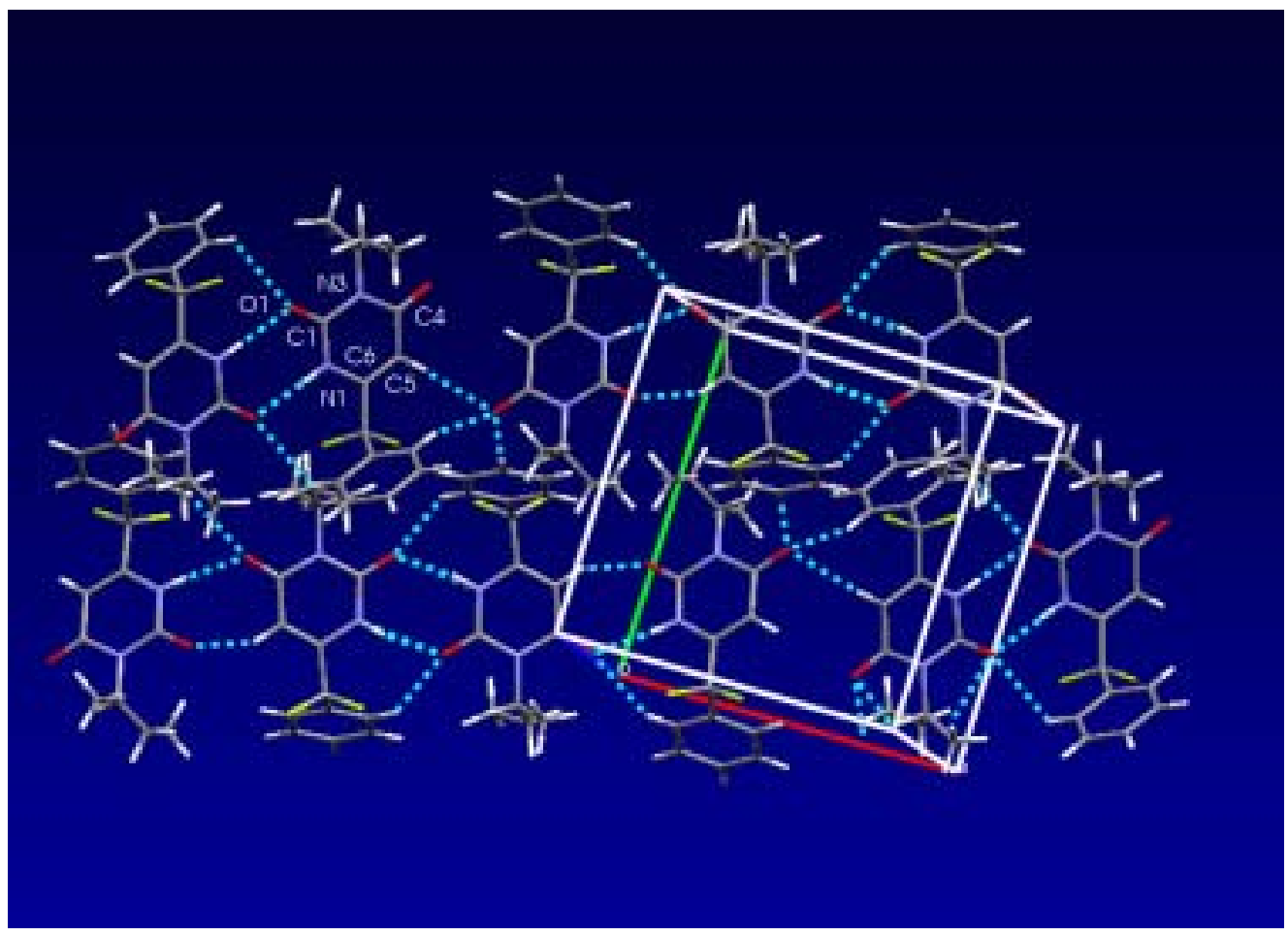

Figure 4. Crystal packing of compound 5e through $\mathrm{N}-\mathrm{H} \cdots \mathrm{O}=\mathrm{C}$ and $\mathrm{C}-\mathrm{H} \cdots \mathrm{O}=\mathrm{C}$ hydrogen bonds. 
6-(Difluoromethylphenyl)-3-ethyl-2,4-pyrimidindione (5f). The crude reaction product was washed with $n$-hexane- $\mathrm{CH}_{2} \mathrm{Cl}_{2}(5: 1)$ to give pure $\mathbf{5 f}$ as a white solid (78\% yield): $\mathrm{mp} 135-137^{\circ} \mathrm{C}$. ${ }^{1} \mathrm{H}$ NMR (300 MHz, $\left.\mathrm{CDCl}_{3}\right) \delta 1.07(\mathrm{t}, J=7.1,3 \mathrm{H}), 3.85(\mathrm{c}, J=7.0,2 \mathrm{H}), 5.74(\mathrm{~s}, 1 \mathrm{H}), 7.32-7.35(\mathrm{~m}, 3 \mathrm{H})$, 7.47-7.50 (m, 2H); ${ }^{13} \mathrm{C}$ NMR (75.5 MHz, $\left.\mathrm{CDCl}_{3}\right) \delta 13.4(\mathrm{c}), 37.0(\mathrm{t}), 96.6\left(\mathrm{t},{ }^{3} J_{\mathrm{CF}}=4.9\right), 119.4\left(\mathrm{t},{ }^{1} J_{\mathrm{CF}}\right.$ $=243.9), 127.1\left(\mathrm{t},{ }^{3} J_{\mathrm{CF}}=5.7\right), 129.8(\mathrm{~d}), 131.7(\mathrm{~d}), 137.5\left(\mathrm{t},{ }^{2} J_{\mathrm{CF}}=27.3\right), 160.2(\mathrm{~s}), 160.9\left(\mathrm{t},{ }^{2} J_{\mathrm{CF}}=\right.$ 30.0), 168.5 (s); ${ }^{19} \mathrm{~F}$ NMR (282 MHz, $\left.\mathrm{CDCl}_{3}\right) \delta-101.0$ (s, 2F). HRMS calcd for $\mathrm{C}_{13} \mathrm{H}_{12} \mathrm{~F}_{2} \mathrm{~N}_{2} \mathrm{O}_{2}$ 266.0866, found 266.0864.

(+)-(S)-6-(Difluoromethylphenyl)-3-(1-phenylethyl)-2,4-pyrimidindione $\quad(5 \mathrm{~g})$. The crude reaction product was washed with $n$-hexane- $\mathrm{CH}_{2} \mathrm{Cl}_{2}$ (5:1) to give pure $\mathbf{5 g}$ as a white solid (90\% yield): mp $168-170^{\circ} \mathrm{C} .[\alpha]^{25} \mathrm{D}+168.5^{\circ}\left(c 1.04, \mathrm{CHCl}_{3}\right) .{ }^{1} \mathrm{H} \mathrm{NMR}\left(300 \mathrm{MHz}, \mathrm{CDCl}_{3}\right) \delta 1.76(\mathrm{~d}, J=7.2,3 \mathrm{H})$, $5.78(\mathrm{~s}, 1 \mathrm{H}), 6.15(\mathrm{c}, J=7.1,1 \mathrm{H}), 7.15-7.22(\mathrm{~m}, 3 \mathrm{H}), 7.30-7.34(\mathrm{~m}, 4 \mathrm{H}), 7.38-7.45(\mathrm{~m}, 3 \mathrm{H}), 10.7(\mathrm{~s}$, $1 \mathrm{H}) ;{ }^{13} \mathrm{C}$ NMR $\left(75.5 \mathrm{MHz}, \mathrm{CDCl}_{3}\right) \delta 15.9$ (c), 50.7 (d), $100.9\left(\mathrm{t},{ }^{3} J_{\mathrm{CF}}=4.9\right), 116.5\left(\mathrm{t},{ }^{1} J_{\mathrm{CF}}=246.8\right)$, $125.9\left(\mathrm{t},{ }^{3} J_{\mathrm{CF}}=6.0\right), 127.7(\mathrm{~d}), 127.9(\mathrm{~d}), 128.5(\mathrm{~d}), 129.4(\mathrm{~d}), 131.7(\mathrm{~d}), 133.2\left(\mathrm{t},{ }^{2} J_{\mathrm{CF}}=26.7\right), 139.8$ $(\mathrm{s}), 147.4\left(\mathrm{t},{ }^{2} J_{\mathrm{CF}}=33.3\right), 152.7(\mathrm{~s}), 163.1(\mathrm{~s}) ;{ }^{19} \mathrm{~F}$ NMR $\left(282 \mathrm{MHz}, \mathrm{CDCl}_{3}\right) \delta-100.4(\mathrm{~s}, 2 \mathrm{~F}) . \mathrm{HRMS}$ calcd for $\mathrm{C}_{13} \mathrm{H}_{12} \mathrm{~F}_{2} \mathrm{~N}_{2} \mathrm{O}_{2}$ 342.1179, found 342.1190.

6-(Difluoromethylphenyl)-3-(4-ethoxycarbonylphenyl)-2,4-pyrimidindione (5h). The crude reaction product was washed with $n$-hexane- $\mathrm{CH}_{2} \mathrm{Cl}_{2}$ (5:1) and then subjected to flash chromatography [ $n$-hexane:EtOAc (2:1)] on silica gel to give $\mathbf{5 h}$ as a white solid (75\% yield): $\mathrm{mp} 140-142{ }^{\circ} \mathrm{C} .{ }^{1} \mathrm{H}$ NMR $\left(300 \mathrm{MHz}, \mathrm{CDCl}_{3}\right) \delta 1.37(\mathrm{t}, J=7.1,3 \mathrm{H}), 4.38(\mathrm{c}, J=7.1,2 \mathrm{H}), 5.82(\mathrm{~s}, 1 \mathrm{H}), 7.34(\mathrm{~d}, J=8.5,2 \mathrm{H})$, 7.54-7.57 (m, 3H), 7.68-7.71 (m, 2H), $8.02(\mathrm{~d}, J=8.5,2 \mathrm{H}) ;{ }^{13} \mathrm{C} \mathrm{NMR}\left(75.5 \mathrm{MHz}, \mathrm{CDCl}_{3}\right) \delta 14.5$ (c), $61.0(\mathrm{t}), 93.9(\mathrm{~d}), 118.3\left(\mathrm{t},{ }^{1} J_{\mathrm{CF}}=243.9\right), 125.9\left(\mathrm{t},{ }^{3} J_{\mathrm{CF}}=6.0\right), 128.2\left(\mathrm{t},{ }^{2} J_{\mathrm{CF}}=31.0\right), 128.5(\mathrm{~d}), 128.7$ (d), 129.6 (d), 129.8 (d), 130.6 (d), 135.9 (t, $\left.{ }^{2} J_{\mathrm{CF}}=27.0\right), 143.0$ (s), 156.7 (s), 165.0 (s), 165.8 (s); ${ }^{19} \mathrm{~F}$ NMR (282 MHz, $\left.\mathrm{CDCl}_{3}\right) \delta$-98.3 (s, 2F). HRMS calcd for $\mathrm{C}_{20} \mathrm{H}_{16} \mathrm{~F}_{2} \mathrm{~N}_{2} \mathrm{O}_{4} 386.1078$, found 386.1072.

Ethyl [4-(difluoromethylphenyl)-2,6-dioxo-3,6-dihydropyrimidin-1-yl]-acetate (5i). Flash chromatography [ $n$-hexane:EtOAc $(2: 1)]$ on silica gel of the washed product from $n$-hexane- $\mathrm{CH}_{2} \mathrm{Cl}_{2}$ (5:1) gave 5i as a white solid $\left(73 \%\right.$ yield): $\mathrm{mp} 98-100{ }^{\circ} \mathrm{C} .{ }^{1} \mathrm{H} \mathrm{NMR}\left(300 \mathrm{MHz}, \mathrm{CDCl}_{3}\right) \delta 1.15(\mathrm{t}, J=$ 7.2, 3H), 4.07 (c, $J=7.1,2 \mathrm{H}), 4.54(\mathrm{~s}, 2 \mathrm{H}), 5.79(\mathrm{~s}, 1 \mathrm{H}), 7.34-7.35(\mathrm{~m}, 3 \mathrm{H}), 7.49-7.52(\mathrm{~m}, 2 \mathrm{H}) ;{ }^{13} \mathrm{C}$ NMR (75.5 MHz, $\left.\mathrm{CDCl}_{3}\right) \delta 14.8(\mathrm{c}), 43.3(\mathrm{t}), 62.7(\mathrm{t}), 95.9\left(\mathrm{t},{ }^{3} J_{\mathrm{CF}}=5.2\right), 119.5\left(\mathrm{t},{ }^{1} J_{\mathrm{CF}}=243.9\right), 127.2$ $\left(\mathrm{t},{ }^{3} J_{\mathrm{CF}}=6.0\right), 129.8(\mathrm{~d}), 131.7(\mathrm{~d}), 137.5\left(\mathrm{t},{ }^{2} J_{\mathrm{CF}}=27.3\right), 160.1(\mathrm{~s}), 162.0\left(\mathrm{t},{ }^{2} J_{\mathrm{CF}}=28.7\right), 168.2(\mathrm{~s})$, 170.9 (s); ${ }^{19} \mathrm{~F}$ NMR (282 $\left.\mathrm{MHz}, \mathrm{CDCl}_{3}\right) \delta-101.0$ (s, 2F). HRMS calcd for $\mathrm{C}_{15} \mathrm{H}_{14} \mathrm{~F}_{2} \mathrm{~N}_{2} \mathrm{O}_{4} \quad 324.0921$, found 324.0928 . 
6-(Difluoromethylphenyl)-3-(4-fluorophenyl)-2-tioxo-2,3-dihydropyrimidin-4-one (5j). The crude reaction product was washed with $n$-hexane- $\mathrm{CH}_{2} \mathrm{Cl}_{2}(5: 1)$ to give pure $\mathbf{5 j}$ as a yellowish solid (64\% yield): $\mathrm{mp} 176-178^{\circ} \mathrm{C} .{ }^{1} \mathrm{H} \mathrm{NMR}\left(300 \mathrm{MHz} \mathrm{CDCl}_{3}\right) \delta 6.07$ (s, 1H), 7.08-7.11 (m, 4H), 7.46$7.49(\mathrm{~m}, 3 \mathrm{H}), 7.60-7.61(\mathrm{~m}, 2 \mathrm{H}) ;{ }^{13} \mathrm{C} \mathrm{NMR}\left(75.5 \mathrm{MHz}, \mathrm{CDCl}_{3}\right) \delta 104.8\left(\mathrm{t},{ }^{3} J_{\mathrm{CF}}=5.5\right), 117.5\left(\mathrm{~d},{ }^{2} J_{\mathrm{CF}}=\right.$ 23.6), $118.1\left(\mathrm{t},{ }^{1} J_{\mathrm{CF}}=247.1\right), 127.4\left(\mathrm{t},{ }^{3} J_{\mathrm{CF}}=5.7\right), 130.1\left(\mathrm{t},{ }^{2} J_{\mathrm{CF}}=28.4\right), 130.5(\mathrm{~d}), 132.0\left(\mathrm{~d},{ }^{3} J_{\mathrm{CF}}=9.2\right)$, $133.1(\mathrm{~d}), 134.6\left(\mathrm{t},{ }^{2} J_{\mathrm{CF}}=26.1\right), 136.6\left(\mathrm{~d},{ }^{4} J_{\mathrm{CF}}=3.4\right), 163.1(\mathrm{~s}), 164.3\left(\mathrm{~d},{ }^{1} J_{\mathrm{CF}}=246.6\right), 180.5(\mathrm{~s}) ;{ }^{19} \mathrm{~F}$ NMR (282 MHz, $\left.\mathrm{CDCl}_{3}\right) \delta-99.2(\mathrm{~s}, 2 \mathrm{~F}),-115.9(\mathrm{~m}, 1 \mathrm{~F})$. HRMS calcd for $\mathrm{C}_{17} \mathrm{H}_{11} \mathrm{~F}_{3} \mathrm{~N}_{2} \mathrm{OS} 348.0544$, found 348.0544 .

\section{6-(Difluoromethylphenyl)-3-(4-methylphenyl)-2-tioxo-2,3-dihydropyrimidin-4-one}

$(5 \mathbf{k})$.

The crude reaction product was washed with $n$-hexane- $\mathrm{CH}_{2} \mathrm{Cl}_{2}(5: 1)$ to give pure $\mathbf{5 k}$ as a yellowish solid (68\% yield): mp 180-182 ${ }^{\circ} \mathrm{C} .{ }^{1} \mathrm{H}$ NMR (300 MHz, $\left.\mathrm{CDCl}_{3}\right) \delta 2.29$ (s, 3H), 6.09 (s, 1H), 6.97 (d, J $=8.1,2 \mathrm{H}), 7.18(\mathrm{~d}, J=8.1,2 \mathrm{H}), 7.47-7.65(\mathrm{~m}, 5 \mathrm{H}) ;{ }^{13} \mathrm{C} \mathrm{NMR}\left(75.5 \mathrm{MHz}, \mathrm{CDCl}_{3}\right) \delta 21.9(\mathrm{c}), 105.1(\mathrm{t}$, $\left.{ }^{3} J_{\mathrm{CF}}=5.5\right), 118.2\left(\mathrm{t},{ }^{1} J_{\mathrm{CF}}=245.1\right), 127.2\left(\mathrm{t},{ }^{3} J_{\mathrm{CF}}=6.0\right), 129.8(\mathrm{~d}), 130.3\left(\mathrm{t},{ }^{2} J_{\mathrm{CF}}=30.4\right), 130.7(\mathrm{~d}), 131.5$ (d), $133.3(\mathrm{~s}), 134.7$ (t, $\left.{ }^{2} J_{\mathrm{CF}}=26.4\right), 138.2$ (s), 140.1 (s), 162.8 (s), 180.5 (s); ${ }^{19} \mathrm{~F}$ NMR (282 MHz, $\left.\mathrm{CDCl}_{3}\right) \delta-98.4(\mathrm{~s}, 2 \mathrm{~F})$. HRMS calcd for $\mathrm{C}_{18} \mathrm{H}_{14} \mathrm{~F}_{2} \mathrm{~N}_{2} \mathrm{OS} 344.0794$, found 344.0778.

6-(Difluoromethylphenyl)-3-(4-nitrophenyl)-2-tioxo-2,3-dihydropyrimidin-4-one (5l). The crude reaction product was washed with $n$-hexane- $\mathrm{CH}_{2} \mathrm{Cl}_{2}$ (5:1) and then subjected to flash chromatography [ $n$-hexane:EtOAc (2:1)] on silica gel to give $\mathbf{5 l}$ as a yellowish solid (70\% yield): mp 187-189 ${ }^{\circ}$. ${ }^{1} \mathrm{H}$ NMR (300 MHz, $\left.\mathrm{CDCl}_{3}\right) \delta 6.06$ (s, 1H), 7.19-7.24 (m, 5H), 7.37-7.40 (m, 2H), 7.91$7.94(\mathrm{~m}, 2 \mathrm{H}) ;{ }^{13} \mathrm{C} \mathrm{NMR}\left(75.5 \mathrm{MHz}, \mathrm{CDCl}_{3}\right) \delta 103.9\left(\mathrm{t},{ }^{3} J_{\mathrm{CF}}=5.7\right), 116.5\left(\mathrm{t},{ }^{1} J_{\mathrm{CF}}=246.0\right), 124.8(\mathrm{~d})$, $126.4\left(\mathrm{t},{ }^{3} J_{\mathrm{CF}}=5.5\right), 129.2(\mathrm{~d}), 130.9(\mathrm{~d}), 131.9(\mathrm{~d}), 133.2\left(\mathrm{t},{ }^{2} J_{\mathrm{CF}}=26.4\right), 145.3(\mathrm{~s}), 147.5(\mathrm{~s}), 148.0(\mathrm{t}$, $\left.{ }^{2} J_{\mathrm{CF}}=33.6\right), 160.3(\mathrm{~s}), 178.1(\mathrm{~s}) ;{ }^{19} \mathrm{~F} \mathrm{NMR}\left(282 \mathrm{MHz}, \mathrm{CDCl}_{3}\right) \delta-95.7(\mathrm{~s}, 2 \mathrm{~F})$. HRMS calcd for $\mathrm{C}_{17} \mathrm{H}_{11} \mathrm{~F}_{2} \mathrm{~N}_{3} \mathrm{O}_{3} \mathrm{~S}$ 375.0489, found 375.0463.

6-(Difluoromethylphenyl)-3-ethyl-2-tioxo-2,3-dihydropyrimidin-4-one $\quad(5 \mathrm{~m})$. The crude reaction product was washed with $n$-hexane- $\mathrm{CH}_{2} \mathrm{Cl}_{2}$ (5:1) and then subjected to flash chromatography [ $n$-hexane:EtOAc (2:1)] on silica gel to give $\mathbf{5 m}$ as a yellowish solid (66\% yield): $\mathrm{mp} 110-112{ }^{\circ} \mathrm{C} .{ }^{1} \mathrm{H}$ NMR (300 MHz, $\left.\mathrm{CDCl}_{3}\right) \delta 1.16(\mathrm{t}, J=7.0,3 \mathrm{H}), 4.42(\mathrm{c}, J=6.3,2 \mathrm{H}), 6.05(\mathrm{~s}, 1 \mathrm{H}), 7.29-7.47$ (m, 5H); ${ }^{13} \mathrm{C} \mathrm{NMR}\left(75.5 \mathrm{MHz}, \mathrm{CDCl}_{3}\right) \delta 12.8(\mathrm{c}), 43.5(\mathrm{t}), 102.1(\mathrm{~d}), 119.3\left(\mathrm{t},{ }^{1} J_{\mathrm{CF}}=243.9\right), 127.3\left(\mathrm{t},{ }^{3} J_{\mathrm{CF}}=5.7\right)$, $129.8(\mathrm{~d}), 131.6(\mathrm{~d}), 137.6\left(\mathrm{t},{ }^{2} J_{\mathrm{CF}}=27.6\right), 159.9\left(\mathrm{t},{ }^{2} J_{\mathrm{CF}}=29.6\right), 166.8(\mathrm{~s}), 182.2(\mathrm{~s}) ;{ }^{19} \mathrm{~F}$ NMR $(282$ $\left.\mathrm{MHz}, \mathrm{CDCl}_{3}\right) \delta-100.8$ (s, 2F). HRMS calcd for $\mathrm{C}_{13} \mathrm{H}_{12} \mathrm{~F}_{2} \mathrm{~N}_{2} \mathrm{OS} 282.0638$, found 282.0635. 
6-(Difluoromethylphenyl)-3-(4-fluorophenyl)-5-methyl-2,4-pyrimidindione (5n). The crude reaction product was washed with $n$-hexane- $\mathrm{CH}_{2} \mathrm{Cl}_{2}(5: 1)$ to give pure $\mathbf{5 n}$ as a white solid (82\% yield): mp $179-181^{\circ} \mathrm{C} .{ }^{1} \mathrm{H}$ NMR $\left(300 \mathrm{MHz}, \mathrm{CDCl}_{3}\right) \delta 1.83(\mathrm{~s}, 3 \mathrm{H}), 7.26-7.28(\mathrm{~m}, 4 \mathrm{H}), 7.57-7.73(\mathrm{~m}, 5 \mathrm{H}) ;{ }^{13} \mathrm{C}$ NMR (75.5 MHz, $\left.\mathrm{CDCl}_{3}\right) \delta 10.9(\mathrm{c}), 103.7(\mathrm{~s}), 115.5\left(\mathrm{t},{ }^{2} J_{\mathrm{CF}}=20.4\right), 115.6\left(\mathrm{~d},{ }^{2} J_{\mathrm{CF}}=22.4\right), 119.2(\mathrm{t}$, $\left.{ }^{1} J_{\mathrm{CF}}=244.3\right), 125.8\left(\mathrm{t},{ }^{3} J_{\mathrm{CF}}=5.2\right), 129.0(\mathrm{~d}), 130.9(\mathrm{~d}), 131.1\left(\mathrm{~d},{ }^{3} J_{\mathrm{CF}}=8.6\right), 134.3\left(\mathrm{~d},{ }^{4} J_{\mathrm{CF}}=2.9\right), 135.7$ $\left(\mathrm{t},{ }^{2} J_{\mathrm{CF}}=27.0\right), 154.6(\mathrm{~s}), 161.4\left(\mathrm{~d},{ }^{1} J_{\mathrm{CF}}=243.1\right), 165.8(\mathrm{~s}) ;{ }^{19} \mathrm{~F}$ NMR $\left(282 \mathrm{MHz}, \mathrm{CDCl}_{3}\right) \delta-90.7(\mathrm{~s}, 2 \mathrm{~F})$, $-116.0\left(\mathrm{t}, J_{\mathrm{FH}}=6.7,1 \mathrm{~F}\right)$. HRMS calcd for $\mathrm{C}_{18} \mathrm{H}_{13} \mathrm{~F}_{3} \mathrm{~N}_{2} \mathrm{O}_{2} 346.0929$, found 346.0928.

6-(Difluoromethylphenyl)-3-(3-trifluoromethylphenyl)-2,4-pyrimidindione (5o). The crude reaction product was washed with $n$-hexane- $\mathrm{CH}_{2} \mathrm{Cl}_{2}$ (5:1) to give pure $\mathbf{5 0}$ as a white solid (79\% yield): mp 158-160 ${ }^{\circ} \mathrm{C} .{ }^{1} \mathrm{H}$ NMR $\left(300 \mathrm{MHz}, \mathrm{CDCl}_{3}\right) \delta 1.72(\mathrm{t}, J=2.3,3 \mathrm{H}), 7.45-7.47(\mathrm{~m}, 4 \mathrm{H}), 7.58-7.64(\mathrm{~m}$, $5 \mathrm{H}) ;{ }^{13} \mathrm{C}$ NMR $\left(75.5 \mathrm{MHz}, \mathrm{CDCl}_{3}\right) \delta 11.2(\mathrm{c}), 110.6(\mathrm{~s}), 119.3\left(\mathrm{t},{ }^{1} J_{\mathrm{CF}}=245.4\right), 126.9\left(\mathrm{c},{ }^{3} J_{\mathrm{CF}}=4.0\right)$, $127.0\left(\mathrm{t},{ }^{3} J_{\mathrm{CF}}=5.2\right), 127.5\left(\mathrm{c},{ }^{3} J_{\mathrm{CF}}=3.8\right), 130.6(\mathrm{~d}), 131.6(\mathrm{~d}), 133.0\left(\mathrm{c},{ }^{2} J_{\mathrm{CF}}=32.8\right), 133.1\left(\mathrm{t},{ }^{4} J_{\mathrm{CF}}=\right.$ 1.6), $134.2(\mathrm{~d}), 135.6\left(\mathrm{t},{ }^{2} J_{\mathrm{CF}}=26.4\right), 137.8(\mathrm{~s}), 144.3\left(\mathrm{t},{ }^{2} J_{\mathrm{CF}}=29.0\right), 152.4(\mathrm{~s}), 166.5(\mathrm{~s})$, (the signal from the $\mathrm{CF}_{3}$ group was obscured due to its low intensity); ${ }^{19} \mathrm{~F}$ NMR $\left(282 \mathrm{MHz}, \mathrm{CDCl}_{3}\right) \delta-63.1$ (s, 3F), -93.1 (s, 2F). HRMS calcd for $\mathrm{C}_{19} \mathrm{H}_{13} \mathrm{~F}_{5} \mathrm{~N}_{2} \mathrm{O}_{2}$ 396.0897, found 396.0891.

6-(Difluoromethylphenyl)-3-(4-fluorophenyl)-5-phenyl-2,4-pyrimidindione $\quad$ (5p). Flash chromatography of the crude reaction product [ $n$-hexane:EtOAc $(2: 1)]$ on deactivated silica gel $(2 \%$ $\mathrm{Et}_{3} \mathrm{~N}$ in hexane) gave 5p as a white solid (85\% yield): mp $210-212{ }^{\circ} \mathrm{C} .{ }^{1} \mathrm{H} \mathrm{NMR}\left(300 \mathrm{MHz}, \mathrm{CDCl}_{3}\right) \delta$ 6.98-7.02 (m, 2H), 7.24-7.36 (m, 5H), 7.42-7.56 (m, 7H), 11.8 (br s, 1H); ${ }^{13} \mathrm{C}$ NMR (75.5 MHz, $\left.\mathrm{CDCl}_{3}\right) \delta 114.0\left(\mathrm{t},{ }^{3} J_{\mathrm{CF}}=1.7\right), 116.0\left(\mathrm{~d},{ }^{2} J_{\mathrm{CF}}=22.9\right), 117.5\left(\mathrm{t},{ }^{1} J_{\mathrm{CF}}=247.1\right), 125.8\left(\mathrm{t},{ }^{3} J_{\mathrm{CF}}=5.2\right), 127.6$ (d), 127.9 (d), 128.9 (d), 131.1 (d), 131.2 (d, $\left.{ }^{3} J_{\mathrm{CF}}=9.2\right), 131.4$ (d), 132.0 (s), $134.4\left(\mathrm{t},{ }^{2} J_{\mathrm{CF}}=26.4\right)$, $142.9\left(\mathrm{t},{ }^{2} J_{\mathrm{CF}}=29.0\right), 150.7(\mathrm{~s}), 160.2(\mathrm{~s}), 162.4\left(\mathrm{~d},{ }^{1} J_{\mathrm{CF}}=216.1\right), 163.5(\mathrm{~s}) ;{ }^{19} \mathrm{~F}$ NMR $(282 \mathrm{MHz}$, $\left.\mathrm{CDCl}_{3}\right) \delta$-87.7 (s, 2F), -114.4 (m, 1F). HRMS calcd for $\mathrm{C}_{23} \mathrm{H}_{15} \mathrm{~F}_{3} \mathrm{~N}_{2} \mathrm{O}_{2}$ 408.1085, found 408.1080.

5-Bromo-6-(difluoromethylphenyl)-3-(4-fluorophenyl)-2,4-pyrimidindione (5q). ${ }^{1}$ The crude reaction product was washed with $n$-hexane- $\mathrm{CH}_{2} \mathrm{Cl}_{2}$ (5:1) and then subjected to flash chromatography [ $n$-hexane:EtOAc $(2: 1)]$ to give $\mathbf{5 q}$ as a yellowish solid (78\% yield): mp $165-167^{\circ} \mathrm{C} .{ }^{1} \mathrm{H}$ NMR $(300$ $\left.\mathrm{MHz}_{\mathrm{CDCl}}\right) \delta$ 7.06-7.21 (m, 4H), 7.40-7.43 (m, 3H), 7.62-7.65 (m, 2H); ${ }^{13} \mathrm{C}$ NMR (75.5 MHz, $\left.\mathrm{CDCl}_{3}\right) \delta 96.7(\mathrm{~s}), 117.4\left(\mathrm{~d},{ }^{2} J_{\mathrm{CF}}=23.0\right), 118.9\left(\mathrm{t},{ }^{1} J_{\mathrm{CF}}=247.1\right), 127.3\left(\mathrm{t},{ }^{3} J_{\mathrm{CF}}=5.2\right), 130.2(\mathrm{~d}), 132.0$

1 The brominated enamino ester necessary for the preparation of $\mathbf{5 q}$ was prepared through NBS bromination of the corresponding enamino ester 8a. See: Fustero, S.; Salavert, E.; Sanz-Cervera, J. F.; Román, R.; Fernández-Gutiérrez, B.; Asensio, A. Lett. Org. Chem. 2004, 1, 93-98. 
$\left(\mathrm{d},{ }^{3} J_{\mathrm{CF}}=8.6\right), 132.7(\mathrm{~d}), 133.4\left(\mathrm{~d},{ }^{4} J_{\mathrm{CF}}=3.4\right), 135.2\left(\mathrm{t},{ }^{2} J_{\mathrm{CF}}=26.1\right), 150.1\left(\mathrm{t},{ }^{2} J_{\mathrm{CF}}=29.0\right), 153.8(\mathrm{~s})$, $163.0(\mathrm{~s}), 164.3\left(\mathrm{~d},{ }^{1} J_{\mathrm{CF}}=246.0\right) ;{ }^{19} \mathrm{~F}$ NMR $\left(282 \mathrm{MHz}, \mathrm{CDCl}_{3}\right) \delta-94.6(\mathrm{~s}, 2 \mathrm{~F}),-115.6(\mathrm{~m}, 1 \mathrm{~F})$. HRMS calcd for $\mathrm{C}_{17} \mathrm{H}_{10} \mathrm{BrF}_{3} \mathrm{~N}_{2} \mathrm{O}_{2}$ 409.9877, found 409.9872 .

6-[(1-Naphtyl)difluoromethyl)]-3-(4-fluorophenyl)-2,4-pyrimidindione $\quad \mathbf{( 5 r )}$. The crude reaction product was washed with $n$-hexane- $\mathrm{CH}_{2} \mathrm{Cl}_{2}$ (5:1) and then subjected to flash chromatography [ $n$-hexane:EtOAc (2:1)] to give pure $\mathbf{5 r}$ as a white solid (64\% yield): mp 205-207 ${ }^{\circ} \mathrm{C} .{ }^{1} \mathrm{H}$ NMR (300 $\left.\mathrm{MHz}, \mathrm{CDCl}_{3}\right) \delta 5.90(\mathrm{~s}, 1 \mathrm{H}), 7.06-7.14(\mathrm{~m}, 4 \mathrm{H}), 7.36-7.42(\mathrm{~m}, 1 \mathrm{H})$, 7.50-7.54 (m, 2H), 7.74-7.77 (m, $1 \mathrm{H}), 7.86-7.98(\mathrm{~m}, 3 \mathrm{H}) ;{ }^{13} \mathrm{C} \mathrm{NMR}\left(75.5 \mathrm{MHz}, \mathrm{CDCl}_{3}\right) \delta 100.8\left(\mathrm{t},{ }^{3} J_{\mathrm{CF}}=5.2\right), 115.5\left(\mathrm{~d},{ }^{2} J_{\mathrm{CF}}=23.0\right)$, $116.2\left(\mathrm{t},{ }^{1} J_{\mathrm{CF}}=243.7\right), 122.9\left(\mathrm{t},{ }^{4} J_{\mathrm{CF}}=2.9\right), 123.4(\mathrm{~d}), 124.9\left(\mathrm{t},{ }^{3} J_{\mathrm{CF}}=8.9\right), 125.3\left(\mathrm{t},{ }^{2} J_{\mathrm{CF}}=23.6\right), 125.7$ (d), 126.9 (d), 128.1 (s), 128,3 (d), $128.5\left(\mathrm{~d},{ }^{4} J_{\mathrm{CF}}=3.4\right), 129.0$ (d, $\left.{ }^{3} J_{\mathrm{CF}}=8.6\right), 132.1$ (d), 133.1 (s), $145.6\left(\mathrm{t},{ }^{2} J_{\mathrm{CF}}=31.6\right), 150.1(\mathrm{~s}), 161.3(\mathrm{~s}), 161.6\left(\mathrm{~d},{ }^{1} J_{\mathrm{CF}}=248.9\right) ;{ }^{19} \mathrm{~F}$ NMR $\left(282 \mathrm{MHz}, \mathrm{CDCl}_{3}\right) \delta-$ 101.6 (s, 2F), -117.4 (m, 1F). HRMS calcd for $\mathrm{C}_{21} \mathrm{H}_{13} \mathrm{~F}_{3} \mathrm{~N}_{2} \mathrm{O}_{2}$ 382.0929, found 382.0941.

3-(4-Fluorophenyl)-6-pentadecafluoroheptil-2,4-pyrimidindione (5s). The crude reaction product was washed with $n$-hexane- $\mathrm{CH}_{2} \mathrm{Cl}_{2}$ (5:1) to give pure $\mathbf{5 s}$ as a white solid (82\% yield): $\mathrm{mp}$ 195-197 ${ }^{\circ} \mathrm{C} .{ }^{1} \mathrm{H}$ NMR $\left(300 \mathrm{MHz}, \mathrm{CDCl}_{3}\right) \delta 6.14(\mathrm{~s}, 1 \mathrm{H}), 7.33-7.36(\mathrm{~m}, 4 \mathrm{H}) ;{ }^{13} \mathrm{C}$ NMR $(75.5 \mathrm{MHz}$, $\left.\mathrm{CDCl}_{3}\right) \delta 102.0(\mathrm{~d}), 115.8\left(\mathrm{~d},{ }^{2} J_{\mathrm{CF}}=22.3\right), 131.1\left(\mathrm{~d},{ }^{3} J_{\mathrm{CF}}=8.6\right), 160.0(\mathrm{~s}), 163.3(\mathrm{~s})$, (the signals from the $\mathrm{C}_{7} \mathrm{~F}_{15}$ group were obscured due to their low intensity); ${ }^{19} \mathrm{~F} \mathrm{NMR}\left(282 \mathrm{MHz}, \mathrm{CDCl}_{3}\right)-80.6(\mathrm{~m}, 3 \mathrm{H})$, $-115.2(\mathrm{~s}, 1 \mathrm{~F}),-115.8\left(\mathrm{t}, J_{\mathrm{FF}}=11.3,2 \mathrm{H}\right),-121.7(\mathrm{~s}, 4 \mathrm{H}), 122.2(\mathrm{~s}, 2 \mathrm{H}), 122.9(\mathrm{~s}, 2 \mathrm{H}), 126.9(\mathrm{~s}, 2 \mathrm{H})$. HRMS calcd for $\mathrm{C}_{17} \mathrm{H}_{6} \mathrm{~F}_{16} \mathrm{~N}_{2} \mathrm{O}_{2} 574.0173$, found 574.0159.

3-Phenyl-6-pentadecafluoroheptil-2,4-pyrimidindione (5t). The crude reaction product was washed with $n$-hexane- $\mathrm{CH}_{2} \mathrm{Cl}_{2}$ (5:1) to give pure $\mathbf{5 t}$ as a white solid (84\% yield): $\mathrm{mp} 192-194{ }^{\circ} \mathrm{C} .{ }^{1} \mathrm{H}$ NMR (300 MHz, $\left.\mathrm{CDCl}_{3}\right) \delta 5.70(\mathrm{~s}, 1 \mathrm{H}), 7.13-7.16(\mathrm{~m}, 2 \mathrm{H}), 7.32-7.47(\mathrm{~m}, 3 \mathrm{H}) ;{ }^{13} \mathrm{C} \mathrm{NMR}(75.5 \mathrm{MHz}$, $\left.\mathrm{CDCl}_{3}\right) \delta 95.0$ (d), 126.9 (d), 128.6 (d), 129.2 (d), 138.8 (s), 158.0 (s), 165.4 (s), (the signals from the $\mathrm{C}_{7} \mathrm{~F}_{15}$ group were obscured due to their low intensity); ${ }^{19} \mathrm{~F}$ NMR $\left(282 \mathrm{MHz}, \mathrm{CDCl}_{3}\right)-80.7(\mathrm{~m}, 3 \mathrm{H})$, $115.9\left(\mathrm{t}, J_{\mathrm{FF}}=97.6,2 \mathrm{H}\right),-121.6-(-121.8)(\mathrm{m}, 4 \mathrm{H}), 122.2(\mathrm{~m}, 2 \mathrm{H}), 122.9(\mathrm{~m}, 2 \mathrm{H}), 126.1-(-126.2)(\mathrm{m}$, $2 \mathrm{H})$. HRMS calcd for $\mathrm{C}_{17} \mathrm{H}_{7} \mathrm{~F}_{15} \mathrm{~N}_{2} \mathrm{O}_{2} 556.0268$, found 556.0290.

\section{Preparation of acetylated Wang resin 11.}

Acetic anhidride $(2.5 \mathrm{mmol})$ and pyridine $(2.5 \mathrm{mmol})$ were added dropwise to a suspension of Wang resin 10 (0.83mmol) in $\mathrm{CH}_{2} \mathrm{Cl}_{2}(5 \mathrm{ml})$,. The mixture was stirred for $15 \mathrm{~h}$ and then filtered and 
washed with DMF $(3 \times 5 \mathrm{ml}), \mathrm{CH}_{2} \mathrm{Cl}_{2}(3 \times 5 \mathrm{ml})$, and $\mathrm{MeOH}(3 \times 5 \mathrm{ml})$. The solvent was then removed under vacuum.

Acetylated Wang resin (11). Yellowish solid. IR ( $\left.\mathrm{cm}^{-1}\right): 1730$.

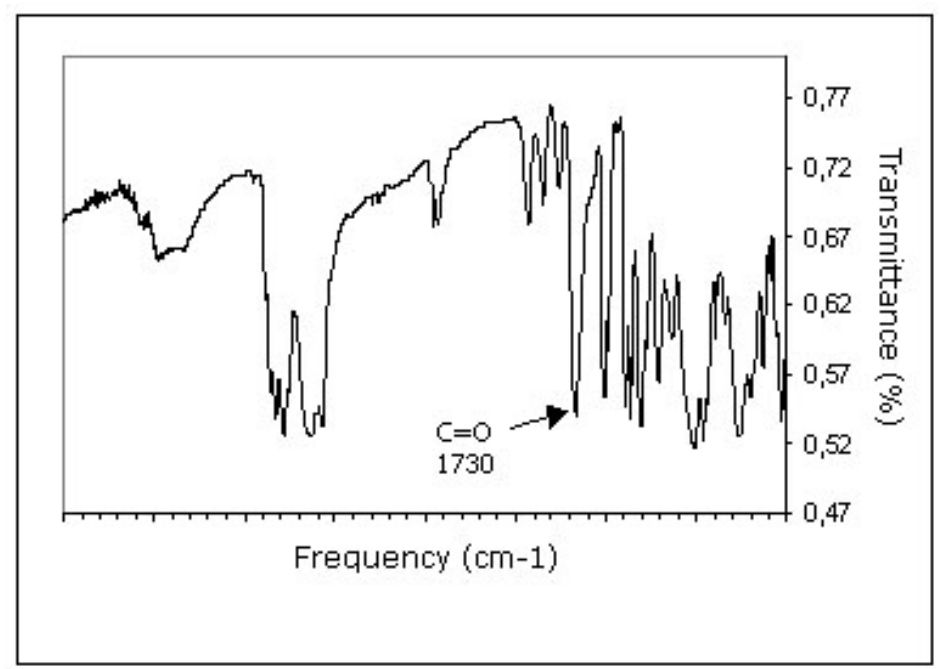

\section{Preparation of the compound resin-bound $\beta$-enaminoester 12.}

$N$-butyllithium (2.5 mmol, 2.5 $\mathrm{M}$ in hexane) was slowly added to a solution of diisopropylamine $(2.4 \mathrm{mmol})$ in $\mathrm{THF}(5 \mathrm{~mL})$ at $0^{\circ} \mathrm{C}$. The mixture was stirred at that temperature for $20 \mathrm{~min}$, after which time the acetilated resin $(0.8 \mathrm{mmol})$ was slowly added. The temperature was maintained at $0^{\circ} \mathrm{C}$ for 1.5 $\mathrm{h}$ more and then a solution of the nitrile $(2.4 \mathrm{mmol})$ in THF $(2 \mathrm{~mL})$ was slowly added dropwise. After $3 \mathrm{~h}$ the reaction was quenched with satd. aq. $\mathrm{NH}_{4} \mathrm{Cl}$ solution and filtered. The resin was washed with DMF $(3 \times 5 \mathrm{ml}), \mathrm{CH}_{2} \mathrm{Cl}_{2}(3 \times 5 \mathrm{ml})$, and $\mathrm{MeOH}(3 \times 5 \mathrm{ml})$ and then dried under vacuum.

Wang resin bound to 3-amino-4,4-difluoro-4-phenyl-2-butenoate (12). Yellow solid. IR $\left(\mathrm{cm}^{-1}\right): 1679,1731,3417,3523$. 


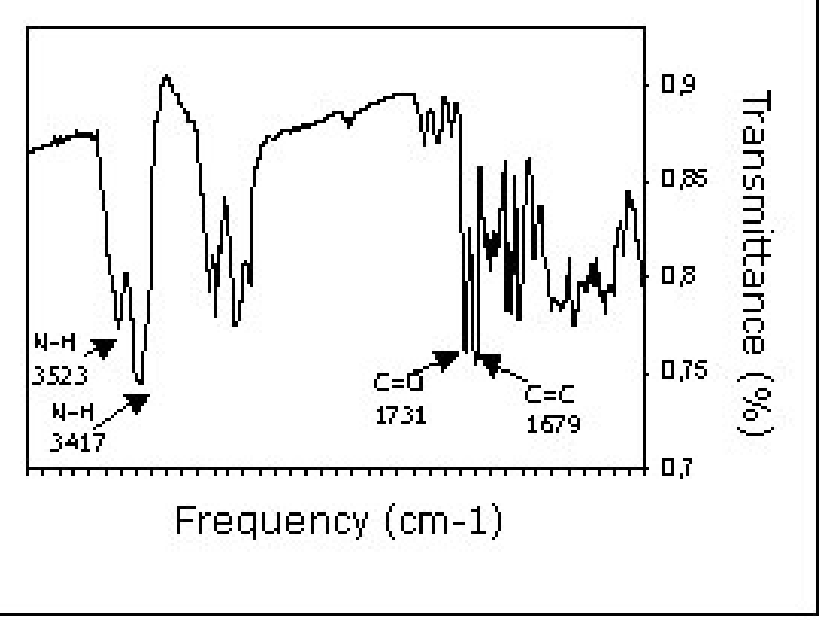

\section{Preparation of compounds 5 from the resin-bound $\beta$-enaminoester 12 .}

General procedure. $\mathrm{NaH}(0.4 \mathrm{mmol})$ was slowly added to a suspension of Wang resin bound to $\beta$-enaminoester $12(0.11 \mathrm{mmol})$ in DMF $(2 \mathrm{ml})$ at $0^{\circ} \mathrm{C}$. The mixture was stirred at that temperature for $1 \mathrm{~h}$ after which time the isocyanate or isotiocyanate $(0.27 \mathrm{mmol})$ was added. After additional stirring at room temperature for $15 \mathrm{~h}$, the reaction was quenched with $\mathrm{H}_{2} \mathrm{O}$, then filtered and washed with $\mathrm{THF} / \mathrm{H}_{2} \mathrm{O}(1: 1)(3 \times 3 \mathrm{ml})$. The aqueous layers were pooled together and extracted with $\mathrm{CH}_{2} \mathrm{Cl}_{2}(3 \times 3$ ml) to remove $\mathrm{CH}_{2} \mathrm{Cl}_{2}$-soluble impurities. The uracils and tiouracils 5 were both soluble in $\mathrm{H}_{2} \mathrm{O}$, but insoluble in $\mathrm{CH}_{2} \mathrm{Cl}_{2}$. The aqueous layer was reduced to a volume of $1 \mathrm{ml}$ under vacuum and then extracted with AcOEt $(3 \times 3 \mathrm{ml})$. The organic layers were pooled together, dried over anh. $\mathrm{Na}_{2} \mathrm{SO}_{4}$, and concentrated to give crude product $\mathbf{5}$, which was purified in the same manner as described above. The uracils and tiouracils obtained from this method were identical in their physical and spectroscopic data to those obtained in solution. 